\title{
Aproximación psicoanalítica a lo político y el discurso del capitalismo
}

\author{
Psychoanalitic approach to politics and \\ the discourse of capitalism
}

\author{
Mario Sergio Kelman ${ }^{1}$ \\ Universidad Nacional de Rosario, Argentina
}

Resumen. El presente texto tiene como objetivo producir un abordaje psicoanalítico del discurso político y el discurso del capitalismo; para formalizar las problemáticas que afectan la subjetividad de la época. El método empleado es la argumentación, arribando a resultados y conclusiones correspondientes al período anual que se informa. Distinguimos la escena de la política de lo real político, manifiesto en el acontecimiento. Cada sistema político se define por el modo de lo sagrado que le es propio. El discurso político clásico sufre una crisis y es sustituido por el discurso del capitalismo, produciendo nuevos efectos en la subjetividad y en el campo social.

Palabras clave. Psicoanálisis, político, discurso, capitalismo, sagrado.

Abstract. The goal of this text is to produce a psychoanalytic approach to political discourse and the discourse of capitalism; to formalize the problems that affect the subjectivity of our time. The argumentative method is used to reach the results and the conclusions for the report of the annual period. We distinguish the political scene of the real political, shown at the event. Every political system is defined by the way of the sacred that is proper. The classic political discourse suffers a crisis and is replaced by the discourse of capitalism, producing new effects on subjectivity and the social field.

Keywords. Psychoanalysis, political, discourse, capitalism, sacred.

\footnotetext{
${ }^{1}$ Mario Sergio Kelman. Consejo de Investigaciones, Universidad Nacional de Rosario, Argentina. Direccion postal: Urquiza 1394 - $2^{\circ}$ piso, oficina “3”, 2000 Rosario (Provincia de Santa Fe), República Argentina. E-mail: mario.kelman@unr.edu.ar
}

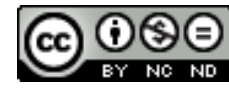




\section{Introducción}

El título del trabajo manifiesta el propósito de este artículo, el realizar un primer abordaje genérico al devenir histórico de lo político a partir del psicoanálisis. Se trata de establecer un conjunto de cuestiones, sin pretender su agotamiento exhaustivo, que serán motivo de nuevas escrituras más particularizadas; de este modo, se deja también al lector, un campo abierto al retrabajo compartido. Se opta por no definir lo político, apoyado en la imposibilidad que ofrece toda definición. La definición intenta en vano, la aprehensión de la esencia; intento siempre fallido por la inadecuación de estructura del lenguaje a lo real.

A diferencia de la propuesta aristotélica de la palabra como etiqueta de la cosa, se dirá que la palabra siempre derrapa la cosa. No obstante, este es un buen punto de partida: la palabra y la cosa.

La política se organiza como discurso, constituyendo su figuración en el espacio de lo representable, ámbito de la inscripción de las subjetividades y de las identificaciones. Lo político es el acontecimiento, cuando la cosa, lo real que escapa siempre a la representación, irrumpe produciendo la ruptura de la escena política y su reformulación, a través de un reordenamiento de la misma. El acontecimiento entonces, excede las intencionalidades.

"Eso" político acontece en una dimensión a-subjetiva. Su elucidación es retroactiva, inferida a través de la aprehensión de las consecuencias, en un despliegue lógico que no es lineal, sino del mismo orden que el acto.

El devenir histórico de lo político implica en consecuencia, una historia que no es cronológica, sino de acontecimientos donde se articulan palabras y cosas, en una superficie imaginaria, una y otra vez. Entonces, ¿cómo leer este devenir que involucra las dimensiones simbólica, imaginaria y real? Por lo pronto, no se trata de la aprehensión de significaciones. Dicha lectura requiere de topologizar la política y lo político, tomando como marco de referencia, el que provee la lógica de un psicoanálisis.
¿Qué es un psicoanálisis sino la lectura de la efectuación de la estructura singular de un ser hablante, trazado en el recorrido de la experiencia del analizante? El analizante hace el recorrido de un análisis, atravesando su realidad hasta arribar al origen imposible, en la escritura de la modalidad singular de goce que le es propia y desconocida, entramada en su síntoma; y en la pregunta por la causa.

Que el origen sea imposible, sitúa una abertura en el plano topológico, en modo análogo al que Freud figura el ombligo de los sueños (Freud, 1900/1984), el autor nos transmite que el análisis de los sueños progresa en la superficie de la escena onírica hasta arribar a un límite, borde del espacio de lo representable que linda con una abertura por donde el ser hablante se umbilica a lo real de la estructura. Es el lugar topológico de donde somos cortados cuando hay nacimiento del sujeto.

De modo similar, la realidad política puesta en plano -y esto es ya una topología- ofrece un cuadro de realidad establecido. La realidad política como dada y arrojada en el plano de la existencia, implica que ya conlleva el montaje de la repetición: lo actual es ya efecto de repetición. La grieta del origen donde se aloja la causa, coincide con lo que se presenta en la ruptura de la escena política: el acontecimiento por el cual irrumpe lo político. La lectura de la política como realidad también hace recorrido.

Un movimiento de la lectura es entonces, el recorrido por el anverso del cuadro de realidad, hasta arribar a la abertura del origen imposible, tanto para la lectura en un psicoanálisis como en la lectura de la escena de la política, efecto de la repetición que inscribe en el futuro lo que retorna del pasado, en un tiempo siempre segundo.

Arribar al límite con la escritura que produce, es un final y al mismo tiempo hay otro recorrido que se abre, por el reverso del cuadro de realidad, donde la abertura de origen no es ya el punto de llegada del recorrido, sino un relanzamiento. Anverso y reverso implican dos recorridos lógico en una heterogeneidad sin identidad, aunque articulados en puntos de atravesamiento, puntos de cruce: no hay simetría ni biunivocidad.

Dos recorridos lógicos que implican lo tercero del corte y del pasaje, con la salvedad que con lo tercero se inicia la cuenta. Lo hétero aquí es alteridad y diferencia. 
Intentaremos hacer este paso de una superficie a la otra, de la política y lo político y el doble recorrido por su anverso y reverso, a través de los hitos que constituyen los nombres de Aristóteles, Agambén, Benjamin y Lacan.

\section{Con Aristóteles. Establecimiento y recorrido por el anverso de la realidad política}

La escritura de Aristóteles tiene miles de años de antigüedad, su letra refleja el esfuerzo ético del filósofo por establecer un orden en la comunidad de su época. Por otra parte implica el gesto que instituye un discurso nuevo, que con Lacan llamamos Discurso Amo o Discurso de Gobernar (Lacan, 1992); el cual materializa el anverso de la realidad política.

Aristóteles (1932) afirma la premisa de una naturaleza política del hombre, que a diferencia del animal, posee un suplemento de lenguaje. Confirma así el estatuto de la política ligada a la disposición de lenguaje y al espacio que el Logos inaugura, propio del ser hablante, que especifica el lazo asociativo con los semejantes de la Polis.

El discurso amo se encausa sobre la imposibilidad de gobernar y produce un lazo social en el modo amoesclavo, que ordena quién gobierna y quién obedece. $\mathrm{La}$ autoridad se inscribe naturalmente en la serie amo, padre, dueño; serie ligada al alma y dotada de la capacidad intelectual de mando. Por otro lado, la serie de aquellos en los que predomina lo corporal y deben natural obediencia; constituida por la mujer, el hijo y el esclavo.

Si la política es natural al hombre, ¿cómo leer aquí el estatuto de la naturaleza? La Naturaleza está signada por la necesidad de conservación del ser viviente, pero ya vida afectada de lenguaje. La Naturaleza se intelige por su despliegue y presentación final, se legitima paradojalmente a partir de su causa teleológica, manifiesta en la finalidad material, como un "finalmente eso es...".

La pregunta se desliza ahora a la necesidad de conservación., la conservación del ser no se referencia en la biología, sino que está tomada en su proyección ontológica y política, redefine la política en una onto-teo-política.

Aristóteles (1932) construye la política como sistema soportado en un cimiento metafísico. Con la conservación del ser en la naturaleza de la política, Aristóteles (1932) anuda política y metafísica, configurando el sostén clásico del proyecto político de Occidente.

La necesidad de conservación excede la necesidad biológica y se desliza al lazo asociativo que se impone a partir de la imposibilidad del ser humano de sostenerse por sí solo. Entonces, un amo que no puede sostenerse por sus propios medios, si no es apelando necesariamente a otros.

Luego, la conservación se liga con la propiedad y con la economía, inscrita como ciencia doméstica en el ámbito familiar; el desarrollo de la economía pasa de la recolección a la producción artesanal y al intercambio, hasta hacer existir las finanzas. Aristóteles (1932) recomienda la medida virtuosa de la economía y el comercio, condenando explícitamente la usura.

A través del estudio comparado de las Constituciones griegas, se explicita su concepción sobre la política como arquitectura de gobierno dispuesta en el espacio topológico de una esfera, donde resalta la cúpula política. La esfera recorta dos espacios. Un espacio interior que aloja lo aceptado cuyo centro y referencia es la virtud; y un espacio exterior que aloja lo rechazado.

Aristóteles (1932) formula la necesidad de justicia y apelaciones éticas en ambos espacios. Anticipa cuestiones valiosas que aún tienen vigencia, como la representatividad equitativa y legítima, la división de poderes, la inclusión equilibrada de los sectores de la comunidad y el carácter de ciudadano que discurre sobre la condición de elector y de elegido.

No obstante, qué lugar para la vida desnuda o zoé, que Agambén diferencia de la bios (Agambén, 2004). Se propone la tesis que la vida desnuda, lo vivo, se localiza en lo rechazado, lo excluido por su ética; el latido vivo de la zoé en el planteo aristotélico, está en el campo de la animalidad. Los goces corporales y la sexualidad; considerados siempre excesivos y teratológicos medidos en términos de temperancia y desvíos de la virtud, son excluidos al espacio exterior de la esfera de la polis.

Finalmente, la formulación aristotélica desemboca en un problema crucial, la soberanía política. Problema para el cual dice no tener respuesta, aunque nos deja el 
aporte de una valiosa pregunta: ¿Soberanía del hombre o soberanía de la ley? En ambos casos no hay respuesta. Una está limitada por la corrupción de los hombres y la otra, por la inaplicabilidad de la ley recubriendo la vida.

Con Agambén. Corte de la superficie topológica, abertura y paso al envés de la política

Agambén lee a Aristóteles y diferencia los dos términos de la lengua griega "zoe" y "bios" que traducimos al castellano como "vida" (Agambén, 2004). Bios es vida tomada en el lenguaje, Logos que define modos de vivir (en plural y sin guiones).

Zoe, la nuda vida, es vida desnuda, desinvestida e irrepresentable, que conlleva el modo-de-vivir que le es propio, inherente al vivir mismo, que proviene de su naturaleza singular. Vida y ser son aquí, términos muy próximos.

Agambén designa la inclusión de la nuda vida en la esfera de la política, como el acontecimiento político de la modernidad, modificando la política clásica. La política se enfoca en el control y el dominio de los cuerpos vivos, deviniendo biopolítica. Dicho acontecimiento se inaugura trágicamente con el surgimiento del nazismo.

Agambén retoma a Aristóteles, haciendo lugar a lo forcluido, lo expulsado por el sistema político clásico. Pero incluido como rechazado y objeto de una segunda inclusión-exclusión constituida por la aplicación de la excepción soberana del Estado de Derecho; para operar sobre ello en un espacio homogéneo, vacuolar e indiferenciado, cuyo paradigma es el campo de concentración. Las diferencias se tramitan por medio de la segregación y la asignación geográfica de lugares y no-lugares; existencia y marginalidad; entes y deshechos.

La topología que soporta la formulación de Agambén no es la esfera sino el cross cap; el cross cap es una esfera modificada por un corte y pegado de la cara interna con la cara externa de la superficie resultante del corte. De modo tal que resulta un espacio esférico clásico coexistiendo con un espacio moebiano; donde lo exterior y lo interior se continúan.

Agambén retoma la formulación clásica de Aristóteles sobre la política, pero reabriendo la hiancia que éste había suturado. El sistema ya no responde a una esfera como forma virtuosa, que ordena y deslinda equilibradamente un interior aceptable y un exterior de lo rechazado.

Agambén presenta un sistema agujereado con superficies torsionadas, que posibilita un pasaje de una interioridad a una exterioridad en comunicación continua, cuyo paso modifica los espacios mismos. Veamos un ejemplo. Las preocupaciones de Aristóteles y Agambén convergen en un tema particular, cual es la soberanía como elemento fundamental de la política.

La cuestión de la soberanía se explica por la propiedad de la decisión como elemento político fundante. Se trata de la decisión soberana, cuyo extremo es el derecho de dar muerte. El soberano está tanto en el interior del sistema como en su exterior: soberano incluido en el espacio de aplicación de la norma, pero también en el exterior, lugar donde exceptuándose, le es posible la decisión de dictar la ley.

Si bien Agambén reconoce explícitamente que la formulación de un soberano coexistiendo en un espacio interior y exterior es una precisión topológica, la propuesta de soportar la lectura de su texto en la figura topológica de un cross cap, es propia e intenta topologizar y mostrar la operación producida en la política moderna.

Agambén recorre la excepción soberana en la elaboración de Carl Schmitt, ideólogo del nazismo; y en el Homo Sacer, figura del derecho romano arcaico que hace visible la nuda vida insacrificable y pasible de muerte impunemente, en una serie donde podría agregarse la marginación social y los devenidos deshechos del capitalismo (Agambén, 2004).

El Estado no se fundaría en el lazo social ni en el contrato o pacto social que nos mantiene en el ámbito del Padre, sino en una instancia más primordial, en la desligadura (Agambén, 2004).

No se trata de la desligadura de un lazo preexistente, sino desligadura original de una vida desnuda arrojada ante la potencia soberana, caracterizada por la potestad de decidir su muerte; situado ahora en un más allá del Padre. Desligadura que no es de ningún lazo, ni persona, ni algo; sino des-ligadura de la excepción. 
Desligadura de la excepción que excluye lo que es comprendido e incluye lo excluido en el mismo pliegue doble; tal es la forma del vínculo soberano con la politización de la vida nuda, como puesta en relación a un poder soberano, instituido por la vida sin amparo, como resto yacente.

La dimensión de lo político alcanzaría de este modo, una dimensión primordial y anterior respecto de la escena de la política, dada por la instauración de la decisión soberana y la nuda vida, punto de diferencia absoluta.

Con Nietzsche. Vaciamiento de la abertura de lo sagrado y babilitación de una condición profana que habilita la praxis política

Primer axioma. La abertura del origen imposible de la estructura, aloja el espacio de lo sagrado, donde la transmisión se articula al discurso.

Segundo axioma. El recorrido de lectura por el reverso de la escena política parte de interrogar qué vierte cada sistema político en el vacío de lo sagrado que es propio a su relato.

- Primero: Vaciamiento nietzscheano de la abertura de lo sagrado.

Nietzsche proclama la muerte de Dios a través de su frase "Dios ha muerto" (Nietzsche, 1882), forma de negación de Dios que hace surgir el vacío, el nihil que usualmente su nombre llena y sutura.

Nietzsche liga la muerte de Dios a un anuncio de la muerte de la metafísica platónica, invierte la perspectiva de una determinante de ideales celestiales y apolíneos, reemplazados por un principio físico, un principio dionisíaco, vital.

El nihilismo es una operación activa y constante que afecta cada sistema, produciendo una nada por la pérdida entrópica que le es inherente. Está acompañado de otra operación que permitela salida de la nada, por la formulación de una nueva posición de valores o transvaloración.

Junto a la transvaloración de valores, constituye un trípode fundamental de su filosofía, con la articulación de la voluntad de poder -carácter fundamental del ser de lo ente- y del ser como el eterno retorno de lo mismo. Voluntad de poder que ha de leerse como un querer puro, sin predicación. Un querer vacío que le es propio, inmanencia del ser.

El eterno retorno de lo mismo. Lo eterno no se define por una duración, ni el retorno se define por un trayecto. Eterno retorno es un repercutirse del ahora. Ritmo, tiempo, latido, lo vivo. La vía de la voluntad de poder es el arte, creación y producción.

Nietzsche reúne en su escritura la formalización de su búsqueda filosófica y de su búsqueda personal. Éste intenta establecer los fundamentos de su filosofía y un soporte personal de su existencia subjetiva. Concibe la producción del arte como extracción de rasgos fundamentales. Es decir, lo escrito como soporte que produce existencia y sostiene lo vivo.

Llegado a esta instancia, su texto vacila entre la vía del arte como escritura y la teoría del valor como magnitud, como número, que desemboca en una elucubración sobre un principio fisiológico del organismo, como determinación. Posiblemente, un intento de regulación del cuerpo y del estado de embriaguez. Vía vacilante que manifiesta el desagregado, la fragmentación del cuerpo que ya afectaba su subjetividad. Al poco tiempo, Nietzsche cae en la locura y su escritura se detiene.

El "Dios ha muerto" establece una nada activa, vaciamiento necesario para una generación creadora, por la vía del arte como escritura que da forma a lo vivo. Nietzsche busca una salida personal ética mediante la escritura de rasgos singulares que fundamentaran su existencia.

Nietzsche a su vez, produce el vaciamiento de lo sagrado y abre simultáneamente una perspectiva profana como condición de una praxis política, que extenderá su incidencia en la producción política posterior. Esta perspectiva habilita el uso y disposición de los medios que en el espacio sagrado, la religión separa y mantiene indisponibles para los humanos, porque pertenecen al orden divino.

- Intermedio. La abertura de lo sagrado en el sistema político y en la experiencia de un psicoanálisis.

¿Puede aproximarse la inexistencia del Otro en el final de análisis con la muerte de Dios? 
Las siguientes reflexiones se referencian en la orientación lacaniana del psicoanálisis; quizás presenten alguna aridez al lector, pero no es posible ahorrar el esfuerzo de lectura que reside en el desciframiento mismo e inevitable del texto. No se trata de la aprehensión de una significación que banalice y neutralice los enunciados, sino del efecto que la resonancia hace pasar en cada uno. Esto ocurre, no sin tiempo.

La existencia de Dios se vincula con la experiencia de lenguaje. En cuanto se siga diciendo, la hipótesis de Dios estará allí. Dios es el Decir, por donde se anuda el dicho y la transmisión del lenguaje con las marcas singulares de cada ser hablante, que resuena en el vacío de la estructura. Transmisión que no es del orden del saber; implica la letra como germen.

Este desarrollo se condensa en el neologismo que acuña Lacan con el término "Dieure" -"Diosir"-, contracción de las voces en lengua francesa "Dieux" -"Dios"- y "Dire" -"Decir"- (Lacan, 1975). Vacío en que se transmite la estructura, donde el Padre del nombre se articula al Nombre del Padre.

El momento previo y correlativo de la tragedia antigua, sitúa a los dioses como manifestación de lo real. El cristianismo consuma la operación de reducir lo divino -como revelación de lo real- al Logos, el Verbo. Con el monoteísmo, y el surgimiento de la tragedia moderna, se produce un retiro de los dioses, barrados y sustituidos por el Dios monoteísta, asumido ahora por lo simbólico.

Allouch establece el "gran relato de la modernidad" como un modo de subsistencia de Dios; un trozo de sí, con la historia (Allouch, 2013). En la experiencia analítica, es el camino inverso: reducción del relato del analizante a la escritura de "lo que no cesa de no escribirse"; producción nueva de un significante primordial-Uno. Escritura que hace acontecimiento e implica el cuerpo.

En el final de análisis, por el levantamiento del sujeto supuesto saber y el despojamiento de las identificaciones, se arriba al punto de inexistencia del Otro y a la salida de la historia y de lo generacional, mediante la construcción de a-topía -presencia hecha de ausencia-.
Se trata de una salida que conserva aquello de lo que se sale, produciendo un lugar nuevo en la estructura, luego de la reversión del toro: extimidad. Allí se pone en juego la función deseo del analista, como máxima distancia entre el objeto "a" como a-topía, ausencia de marca y el S1 -significante Uno- como marca de la ausencia.

Allouch plantea que la salida de la historia resulta en atenerse a un presente (Allouch, 2013), esta afirmación debiera matizarse. Es un presente en movimiento, entre un futuro que proviene del pasado y un pasado que no cesa de proyectarse: allí se articula la causa vacía entre dos movimientos: movimiento de la repetición y movimiento de la traslación del nudo borromeo (Toté, 2012).

Pero no se trata tan sólo de la inscripción simbólica de la función de la falta, sino del Otro como Otro sexo, la Mujer toda -sin tacha- es la otra cara de Dios, por lo cual es necesario en el final de análisis, haber transpuesto una segunda barra, que es lógicamente primera respecto del S (A tachada); cual es La (barrada) Mujer.

El vaciamiento de Dios del campo del Otro se produce con la sexuación, con el pasaje desde Dios, marca de lo divino como prototipo del Nombre del Padre, a la existencia de una mujer no-toda. Se devela así "La Mujer Toda" -si existiera-identificada a Dios.

La barradura recae en Dios-Mujer Toda, realizando su inexistencia, posibilitando una-mujer- no-toda, de la que el hombre espera su castración. Esta operación ocurre en el transcurso de un análisis.

Allouch toma una famosa cita de Lacan del Seminario del 13-4-1976. "Suponer el Nombre-delPadre, por cierto, es Dios. Es allí donde el psicoanálisis, si lo logra, prueba que también se puede prescindir del Nombre del Padre. También se puede prescindir de él a condición de que se sirva de él" (Allouch, 2013). La equivalencia entre suponer el Nombre del Padre y suponer a Dios no debe leerse como afirmar que el Nombre del Padre es Dios.

El Nombre del Padre es lo que resta del padre, aquí padre muerto. Allouch le da el estatuto de un fantasma, nombre fantasmal, espectral; lo que resta de la muerte del padre, muerte de Dios, que corresponde a ese 
primer corte que escribe $S$ (A tachada), es un resto que perdura un tiempo y luego ha de desprenderse, con una segunda barradura, segundo corte: La (tachada) Mujer, que posibilita la significancia y la gramática como salida ética de lo espectral.

Prescindir del nombre del padre es salir de hacer culto religioso de dicho nombre como resto, a condición de servirse de él. Es decir, nombre propio que pasa a ser un nombre común, letra, particularidad del ser hablante con la que es posible un uso, por la vía del síntoma.

El ateísmo resultante de la experiencia analítica no surge de la declaración de la muerte de Dios o de la muerte del Padre, sino de la profanación de lo sagrado (Agambén, 2005). Atreverse a lo sagrado restituyendo un uso de la cosa, haciendo con eso, particular al analizante-analista en la experiencia analítica. Esta vía implica en el final de análisis, un pase del inconsciente a lo público; es decir, la gestación de una política a partir de la naturaleza de lo propio de cada uno.

- Segundo. Lo político. Reverso de la política.

Con Benjamin: "Schuld". Lo que el capitalismo vierte en el espacio vacío de lo sagrado

Partamos del hecho verificable de la tensión e inadecuación entre las formas democráticas del Estado de Derecho contemporáneo y el capitalismo. Lacan interpreta que se ha producido una crisis del discurso del amo clásico, sustituido por el discurso del capitalismo, a partir del surgimiento e imposición de un amo moderno materializado en el mercado. Ello implica una caída del significante del amo clásico; sea cual fuere su semblante (rey, presidente, Ministro, etc.) y del orden político-jurídico que regía el espacio social (Lacan, 1972).

Agregamos que, en tanto algo sea arrojado al espacio vacío de lo sagrado, hace de la política una religión. La política deviene prontamente una onto-teo-política, en tanto se vierta en lo sagrado un Ideal, un nombre o un fundamentalismo.

La pregunta se desliza: ¿qué vierte el capitalismo en el sagrado que le es propio? Esta pregunta, así formulada aborda la realidad política por su reverso, parte de la aprehensión de su núcleo político, en lo sagrado que le es propio. Benjamin formula en forma notable que el capitalismo es una religión, en un fragmento cuyo comentario puntuado transcribimos a continuación (Benjamin, 1985).

En primer lugar, Benjamin define el capitalismo como una religión que se asienta no en una creencia, dogma o teología sino en un culto que satisface preocupaciones, penas e inquietudes que encontraban respuesta en otras religiones de antaño. Sostén ante el desamparo que asume el viviente humano, que afecta y modifica el ser. Esta es la primera de cuatro características del capitalismo como religión de culto.

El segundo rasgo es que es una religión de culto permanente, "sin prisa y sin pausa", esfuerzo de adoración manifiesto y constante que afecta el ser, imprime una forma de vivir que lleva a un destino funesto.

El tercer rasgo, es que no se trata de una religión expiatoria de la culpa, sino una religión que engendra la culpa y la extiende para volverla Universal en un movimiento monstruoso. No sólo abarca a la humanidad sino que incluye a Dios en el mismo destino culpabilizante del humano. Movimiento que persevera hasta el final y anuncia la destrucción como destino. El destino apocalíptico del capitalismo se debe a que éste en su desarrollo consume y atenta contra su propio soporte. De esta manera, ceder al culto del capitalismo requiere de un consentimiento de cada ser humano en el cual se juega la responsabilidad ética de cada uno.

El cuarto rasgo es que en el capitalismo lo que va al lugar de Dios es el cenit de la inculpación, modo de presentación y de velamiento del Dios oscuro al que se hace ofrenda. Un Dios oculto tras el requerimiento de culto, del que se espera la revelación en la hora final.

El corazón de la formulación del capitalismo como religión de Benjamin se soporta en la operación que coloca en el vacío de lo sagrado un rasgo escrito: "SCHULD". "Schuld" puede traducirse como deuda y como culpa. Schuld es el nombre incandescente de la exigencia de sacrificio en lo sagrado, que toma fuerza de Ley de mercado, anulando la legalidad instituida. 
Culpa y deuda como sacrificio exigible, "schuld" se intelige por la referencia lacaniana de la articulación de Kant con Sade (Lacan, 1976): un enunciado sin reciprocidad que se propone como imperativo categórico kantiano haciendo ley con una enunciación performativa.

Así, "Schuld" se inscribe como ley, con la misma lógica que la máxima sadiana de derecho al goce, Éste llama al sacrificio exigible de la extracción de plus de goce o plusvalía, que sostiene la economía del capital.

Parafraseando a Sade, y si fuera posible una tal construcción de una norma cuyo enunciado produce irrisión política, podría entredecirse "Tengo derecho a gozar de tu cuerpo, puede decir quienquiera, y ese derecho lo ejerceré, sin que ningún límite me detenga en el capricho de las exacciones que me venga en ganas saciar en él".

De este modo, se establece que el discurso del capitalismo tiene una arista sadiana que lleva la perversión al espacio social y que conduce a la degradación y a la ruptura de los lazos. Impone al sujeto una condición de objeto al servicio de extracción de plusvalía o plus de goce, rechazando su condición subjetiva, semejante. El capitalismo extrema una tendencia presente en el cristianismo, que absolutiza en cada ámbito la estructura del sacrificio que consuma la separación.

La tesis de Benjamin es que el cristianismo se transforma en capitalismo, luego de un momento en que el capitalismo es parasitario del cristianismo.

El capitalismo extrema y absolutiza la separación que la religión efectúa, pero sin hiancia ni paso entre lo sagrado y lo profano. Se trata de otra modalidad de separación, único proceso de separación que inviste cada cosa humana para dividirla de sí misma, en su conversión en mercancía.

El capitalismo realiza una pura forma de separación sin que haya dos términos que separar. En la mercancía la separación es inherente a la forma del objeto, se escinde en valor de uso y valor de cambio. La mercancía deviene fetiche, con lo cual el valor adquiere otro alcance, y precipita el espacio social productivo en la perversión. Coinciden sacralización y profanación absoluta, sin residuo, pura vacuidad.
Benjamin postula que el capitalismo configura un nuevo espacio a partir de la inclusión de Dios en el destino apocalíptico de lo humano; un espacio de sacrificio exigido, sin exterior. He aquí la topología que sostiene el despliegue del discurso del capitalismo, que extraemos del análisis de Benjamin: una esfera sin espacio exterior.

Todo lo actuado, producido y vivido, se divide en sí mismo, y es desplazado a un espacio sin hiancia, homogéneo, de una esfera separada donde el uso se vuelve imposible. Es la esfera del consumo y del espectáculo, dos caras de una misma imposibilidad de uso.

Si profanar es devolver al uso común lo que fue separado a lo sagrado, la religión capitalista apunta a crear un desecho absolutamente Improfanable. Agambén referencia un conflicto en el siglo XII entre la Curia romana y la orden franciscana, donde se establece un canon teológico del consumo (Agambén, 2005).

Los franciscanos o espirituales reivindican de la regla franciscana, la observancia de una "altísima pobreza" afirmando un uso denominado uso de hecho. Éste está sustraído del campo del Derecho y diferenciado del usufructo de la cosa. El uso de hecho admite un uso desprendido de la propiedad de la cosa y de todo derecho de uso. El uso supone que la sustancia de la cosa quede intacta, mientras que el consumo destruye necesariamente la cosa y niega e imposibilita el uso posterior.

La bula de Juan XXII anticipa la antinomia entre uso y consumo, manifiesta en la sociedad de consumo actual y aporta el paradigma de la imposibilidad de uso sin propiedad. La religión capitalista promueve la división de la cosa como mercancía y como objeto de consumo, elevado a cosa improfanable, en tanto el consumo abole el uso.

En la experiencia analítica, la profanación que habilita un uso cuenta con un sagrado que no exige sacrificio. Este punto concierne a la habilitación de un uso de lo sagrado, de lo transmitido y disponible en cada ser hablante, según su deseo. Por allí el discurso analítico posibilitaría una salida del discurso del capitalismo, a poner a prueba en cada análisis singular. 
El uso de lo (in)disponible define el espacio de la invención singular de cada ser hablante, aquello que constituye la poesía del analizante llevada e incorporada a una forma de vivir. Forma de vivir que está en el vivir mismo. El discurso del capitalismo embragado en un empuje al consumo produce una abolición del uso poético de lo disponible de la cosa.

\section{Con Lacan. El discurso del capitalismo}

La política contemporánea está atravesada por los efectos del surgimiento del discurso del capitalismo potenciado por la ciencia y la técnica. La coyuntura actual se corresponde con la emergencia del mundo como un mercado unificado y global, conocido con el nombre de globalización, que se impone a la legalidad de los Estados de Derecho y los regionalismos.

La posición de Lacan es tomar el capitalismo como un discurso. Existen pocas menciones de Lacan sobre el discurso del capitalismo. El primer anuncio es efectuado en la Conferencia de Milán, convocada con el título Del discurso psicoanalítico (Lacan, 1972). Su tesis de partida es que la realidad se constituye por un baño de lenguaje. Esto es, un juego de significantes que empujan el sentido a la manera de un derrapaje sobre las cosas, ligado al hecho de que hay lalengua.

En tanto se organizan en cuatro modos discursivos - discurso del amo, discurso universitario, discurso de la histérica y discurso analítico- producen cuatro modos de lazos sociales, cuatro modos de relaciones en orden al poder, el saber, a la sexuación y a la propia experiencia analítica.

Entonces, la formalización de discursos supone un trabajo respecto de lalengua. Lalengua es lo real del lenguaje. (Lacan, 1970-1974) Cada discurso se especifica a partir de una imposibilidad que lo encausa. Imposibilidad de gobernar, de educar, de analizar, de una relación/razón sexual.

En el discurso del capitalismo la imposibilidad está removida, por lo cual se define como un falso discurso, que conduce al aislamiento y al empuje al consumo, rechazando el lazo social, el inconsciente y el amor.

Lacan resalta la astucia del discurso del capitalismo, al punto de enfatizar que eso funciona, consume y sin imposibilidad, se consume rápidamente. El funcionamiento del discurso del capitalismo consuma su propio soporte, marcha hacia su crisis cíclica de catástrofe y extinción, encontrando allí su real que hace límite. Este movimiento también se corresponde con la indicación de Benjamin, que el capitalismo como religión conduce a la destrucción como destino.

Por otro lado, el discurso del capitalismo remueve la imposibilidad del sujeto de acceder directamente al goce, a través del consumo. El otro cuenta sólo como fuerza de trabajo, fuente de plus de goce equivalente al objeto. Aunque ni siquiera cuenta como objeto. El discurso del capitalismo instituye una gran fábrica de deshechos, que ni siquiera alcanzan la dignidad de objeto; ello connota un sesgo sadiano y lleva la perversión al campo social.

La mercancía cobra el valor de un objeto fetiche que reniega la castración. La ley del Derecho es aceptada en el movimiento de la renegación, lo cual tiene sus consecuencias. Por la misma razón, se evidencia una inadecuación de estructura entre el discurso del capitalismo y el Estado de Derecho y la Democracia moderna.

Pero decir no hay imposibilidad para la extracción sin medida de goce no es decir que no hay imposible. Ocurre que el imposible (real) no está en su lugar, anclado por el calce Real-Simbólico-Imaginario que apresa la causa. Sin calce, las dimensiones real, simbólico e imaginaria se desanudan, afectando el cuadro de realidad y dejando lo imposible en una la deriva, produciendo irrupciones traumáticas, locura y un estado de debilidad mental generalizado.

Por otro lado, la crisis del discurso del amo clásico impone la caída de los Ideales, las jerarquías, las leyes, el Padre, los semblantes de la ley, las convenciones sociales y de lo simbólico en su conjunto.

El discurso del capitalismo sitúa en lugar del agente, un sujeto desvanecido y afectado por el empuje de la ley del mercado. Imperativo de mercado embragado en el superyó de la época que empuja al goce del consumo impuesto, rechazando incluso las huellas de la fantasía y el deseo singular o las condiciones de amor singulares del ser hablante. 
Advertimos las consecuencias en la subjetividad de la época, en la producción de una nueva casuística, que mencionamos sin explayarnos. Irrupción de angustia no localizada -vulgarmente llamada "pánico"-o más allá de eso, la desafectación de un sujeto anónimo y desvanecido.

Pérdida del síntoma de su valor simbólico en cuanto potencia de metáfora y proliferación del síntoma recostado en su cara real, precipitado en síntomas-actos empujado por la compulsión y la impulsividad. Se abre una serie que comprende adicciones, bulimia, anorexia, violencia, fenómenos psicosomáticos y enfermedades graves, locura y debilidad mental generalizada.

\section{Recomienzo y conclusión}

Diversos autores han establecido una distinción entre la política y lo político. La política se define como realidad política; un sistema discreto y separado, configurado como una red significante ajustado a un Significante Uno, un Ideal, a la que subyace un soporte fantasmático; que posibilita lazos identificatorios y libidinales. Lo político es lo real de la política, que irrumpe en los disloques de la realidad política y se sustrae a la representación, a la escena política.

Para el análisis, las fallas de un sistema político son consideradas como una hiancia causal. Es decir, el análisis político encuentra las determinantes de un sistema político en las fallas del sistema precedente. Este abordaje de la política procede por el anverso de la estructura, asignando preexistencia a una realidad política, ya dada a partir de la antecedencia de la articulación simbólico-imaginaria.

Optamos por mantener otra perspectiva, a partir de la antecedencia de lo real y de lo político, que implica un abordaje de la escena política por su reverso. Se plantea otro análisis político a partir de establecer con qué sagrado cuenta cada sistema político.

Releamos el trayecto efectuado a la luz de esta perspectiva. Iniciamos el recorrido con Aristóteles, que vierte en el espacio de lo sagrado el ser, anudando política y metafísica. Continuamos con Agambén, que retoma el planteo aristotélico, produciendo un doble pliegue, doble inclusión-exclusión que ejemplifica con la excepción soberana y el Homo Sacer, para leer el acontecimiento de la política en la modernidad a nuestros días, como es la inclusión de la nuda vida en un espacio indiferenciado y su transformación en biopolítica.

Luego, Nietzsche, que produce un vaciamiento del espacio de lo sagrado a través del nihilismo, y propone el arte y la escritura como vía ética de salida. Benjamin afirma que el capitalismo tiene el estatuto de una religión de culto que vierte en lo sagrado, la voz "schuld". Deuda y culpa que tradujimos como exigencia de sacrificio, ley del mercado que establece como principio la extracción incesante de plus de goce. Arista sadiana del discurso del capitalismo, que profundizamos con la lectura de Lacan, de Kant con Sade.

La experiencia analítica implica un vaciamiento de lo sagrado y salida del discurso del capitalismo, uno por uno. Se trata de un sagrado que no exige sacrificio, sino que proporciona medios para un saber hacer profano con lo propio, el rasgo particular, el sinthome. Una política de los medios prescindiendo de la finalidad, un hacer que es invención, poesía del analizante incorporada en la forma de vivir misma. La clínica tiene una dimensión no sólo terapéutica, sino también ética y política.

\section{Referencias}

Agambén, G. (2004). Homo Sacer I. España: Pre Textos.

Agambén, G. (2005). Profanaciones. Buenos Aires: Adriana Hidalgo Editora.

Allouch, J. (2013). Prisioneros del gran Otro. La injerencia divina. Buenos Aires: El cuenco de plata.

Aristóteles. (1932). La Política. París: Garnier Hermanos. Recuperado de http://biblio.juridicas.unam.mx/ libros/libro.htm?l=766

Benjamin, W. (1985). El capitalismo como religión. Fragmento. Recuperado de http://www.hojaderuta.org/imagenes/ elcapitalismocomoreligionbenjamin.pdf

Freud, S. (1900/1984). La interpretación de los sueños. Buenos Aires: Amorrortu Editores.

Lacan, J. (1976). Kant con Sade. En Escritos (Vol. 2). Madrid: Editorial Siglo XXI.

Lacan, J. (1970-1974). Radiofonía y Televisión. Barcelona: Editorial Anagrama. 
Lacan, J. (1972). Conferencia de Milan. Recuperado de http:/ / elpsicoanalistalector.blogspot.com.ar/2013/03/ jacques-lacan-del-discurso.html

Lacan, J. (1975). Seminario XXII RSI. Documento inédito.

Lacan, J. (1992). El Seminario Libro XVII. El reverso del psicoanálisis. Buenos Aires: Ed. Paidós.
Nietzsche, F. (1882). El Discurso del Loco. Aforismo 125. Recuperado de http://ateismoparacristianos.blogspot. com.ar/2013/09/el-discurso-del-loco-aforismo-125de-la.html

Toté, S. (2012). Cuerpos y nudos. Recuperado de http:// rephip.unr.edu.ar/handle/2133/2005

Recibido: $1^{\circ}$ de mayo de 2014 Aceptado: $1^{\circ}$ de octubre de 2014 